Turk. J. Math. Comput. Sci.

12(2)(2020) 120-127

(C) MatDer

DOI : $10.47000 /$ tjmcs. 704794

\title{
Investigating A Quadratic Bezier Curve Due to N-C-W and N-Bishop Frames
}

\author{
Hatice Kusak Samanci ${ }^{1, *}$ (D), Muhsin Incesu ${ }^{2}$ iD \\ ${ }^{1}$ Department of Mathematics, Faculty of Science and Art, Bitlis Eren University, 13000, Bitlis, Turkey. \\ ${ }^{2}$ Department of Mathematics Education, Faculty of Education, Mus Alparslan University, 49100, Mus, Turkey.
}

Received: 16-03-2020 • Accepted: 29-10-2020

AвstRact. The purpose of our paper is to investigate N-Bishop frame of the quadratic Bezier curve which is one of the effective methods for computer-aided geometric design (CAGD). Then the N-Bishop curvatures and derivative formulas for quadratics Bezier curve are calculated and give some numeric examples.

2010 AMS Classification: 53A04, 68U07

Keywords: Bezier curves, CAGD, N-C-W frame, N-Bishop frame, Modified frames.

\section{INTRODUCTION}

Bishop frame was developed by L. Bishop in 1975 in order to build a more practical alternative parallel frame without using the second derivative on the curve [1]. Recently, many studies have begun to be done in order to investigate the invariants of the curve via Bishop frame. The Bishop frame has been investigated by many researchers for various special curves such as involute evolute curves, Bertrand curves, helix, slant helix until now. Moreover, S. Yilmaz and his colleagues created a new Bishop alternative frame included the binomial vector instead of the tangent vector, [11]. S. Y1lmaz and et. al. investigated new spherical indicators and some characterizations according to the new type Bishop frame, [11,12]. Much work has been done on the Bishop frame as defined by L.Bishop and S.Y1lmaz. Furthermore, a new alternative frame $\{N, C, W\}$ was defined by Scofield providing a different approach [9]. Uzunoğlu and et. al. used a different approach to curves of constant precession [10]. Then N-Bishop frame was firstly given by Ö. Keskin. et al. by using the new Bishop frame methods in [5]. On the other hand, Pierre Bézier (1910-1999) and a French mathematician Paul de Faget de Casteljau (1930) developed a new design curve called Bézier curves between the years 1958 and 1960. Bezier curves provide simplicity in the design processes because of their controllable usage. There are many articles about Bezier curves. The curvature and torsion of Bezier curves, Casteljau algorithm and some other basics of Bezier curves should be found in the books [2] and [6]. Particularly, Sapidis and his colleagues studied on the curvature of a quadratic Bezier curve [8]. Moreover, Floater examined the derivatives of a Bezier curve [3]. Also, Incesu and Kusak Samanc1 studied on the curvatures and Bishop frame of the Bezier curves [4,7]. The aim of this paper is to investigate a quadratic Bezier curve according to N-Bishop Frame. Firstly, we calculated Serret-Frenet frames of a quadratic Bezier curve, then firstly investigated on an alternative modified frame $\{N, C, W\}$. As a result, we computed N-Bishop frame of a quadratic Bezier curve. At the end of our study, we gave some numeric examples.

*Corresponding Author

Email addresses: hkusak@ beu.edu.tr (H. Kusak Samanci), m.incesu@alparslan.edu.tr (M. Incesu) 


\section{Preliminaries}

As we know from differential geometry, the derivative formula of Serret-Frenet frame in the matrix form can be written by

$$
T^{\prime}(s)=\kappa N(s), N^{\prime}(s)=-\kappa T(s)+\tau B(s), B^{\prime}(s)=-\tau B(s),
$$

where $\kappa$ and $\tau$ are curvatures of the Serret-Frenet frame in Euclidean three space. Furthermore, the Bishop frame is relatively parallel the unit tangent field $T$, and the derivative matrix of the Bishop frame is given by

$$
T^{\prime}(s)=k_{1} N_{1}(s)+k_{2} N_{2}(s), N_{1}^{\prime}(s)=-k_{1} T(s), N_{2}^{\prime}(s)=-k_{2} T(s)
$$

where $k_{1}$ and $k_{2}$ are curvatures of the Bishop frame in Euclidean 3-space, [1]. A new Bishop frame named with type-2 Bishop frame, which the frame is parallel to binomial vector field, developed in 2010 by Y1lmaz et al., and its derivative matrix is described by

$$
N_{1}^{\prime}(s)=-k_{1} B(s), N_{2}^{\prime}(s)=-k_{2} B(s), B^{\prime}(s)=k_{1} N_{1}(s)+k_{2} N_{2}(s),
$$

where $k_{1}$ and $k_{2}$ are curvatures of the type-2 Bishop frame in Euclidean 3-space, [11]. Additionally, another new alternative moving frame along a curve called $\{N, C, W\}$ frame in Euclidean 3-space was defined by Scofield in 1995 , $[9,10]$. The new frame is $\{N, C, N \times C=W\}$, where

$$
N, C=\frac{N^{\prime}}{\left\|N^{\prime}\right\|}, W=\frac{\tau T+\kappa B}{\left\|\kappa^{2}+\tau^{2}\right\|} .
$$

The derivative matrix of the alternative moving frame is given with

$$
N^{\prime}(s)=f(s) C(s), C^{\prime}(s)=-f(s) N(s)+g(s) W(s), W^{\prime}(s)=-g(s) C(s),
$$

where $f=\sqrt{\kappa^{2}+\tau^{2}}$ and $g=\frac{\kappa^{2}(\tau / \kappa)^{\prime}}{\kappa^{2}+\tau^{2}}=\sigma f$ are the differentiable functions, [9, 10]. In 2017, Keskin and Yayli first defined a new kind of Bishop frame named with N-Bishop frame for a normal direction curve which is defined as an integral curve of the principal normal of a curve. The derivative matrix of the N-Bishop frame is computed by

$$
N^{\prime}(s)=k_{1} N_{1}(s)+k_{2} N_{2}(s), N_{1}^{\prime}(s)=-k_{1} N(s), N_{2}^{\prime}(s)=-k_{2} N(s)
$$

see in [5]. On the other hand, Bezier curves were firstly developed by P. Bezier (1910-1999) and P. Casteljau (1930) between the years 1958 and 1960. A Bezier curve is defined by

$$
Q(t)=\sum_{i=0}^{n} Q_{i} B_{i}^{n}(t)
$$

where $B_{i}^{n}(t)=\left(\begin{array}{c}n \\ i\end{array}\right) t^{i}(1-t)^{n-i}$ are Bernstein polynomials and $Q_{0}, Q_{1}, \ldots, Q_{n}$ are the control points for each $t \in[0,1]$. The derivatives of the Bezier curves are calculated with

$$
\frac{\mathrm{d}^{r} b^{n}}{\mathrm{~d} t^{r}}(\mathrm{t})=\frac{n !}{(n-r) !} \sum_{\mathrm{i}=0}^{n-r} \Delta^{r} b_{i} \cdot B_{\mathrm{i}}^{\mathrm{n}-\mathrm{r}}(\mathrm{t})
$$

where $\Delta^{r} b_{i}=\sum_{\mathrm{j}=0}^{\mathrm{r}}\left(\begin{array}{c}r \\ j\end{array}\right)(-1)^{\mathrm{r}-\mathrm{j}} b_{\mathrm{i}+\mathrm{j}}$. A Bezier curve which is defined by the starting point $t=0$ and finishing point $t=1$ are tangent to the control polygon at these endpoints. The endpoints of the Bezier curve achieve to manipulate all of the control points. Hence the starting and finishing points are enough to control the whole curve. Many studies have been done about the Bezier curves used in the computer modeling. The basic concepts of these curves can be seen in [2,4,6,7]. Moreover, the de Casteljau algorithm in Farin 1996 and Marsh 2006 provides a technique to evaluate all the points on a Bezier curve that corresponds to the parameter value $t \in[0,1]$. In the Casteljau algorithm, the intermediate points can be found as

$$
Q_{i, k}(t)=\sum_{j=0}^{k} B_{j, k}(t) Q_{i+j}
$$

and the following properties are satisfied

$$
Q_{i, 0}=Q_{i}, Q_{0, n}(t)=Q(t), Q_{i, k}(t)=(1-t) Q_{i, k-1}+t Q_{i+1, k-1} .
$$


The intermediate control points in Casteljau algorithm are

$$
\begin{array}{lllll}
Q_{0,0} & Q_{1,0} & \ldots & Q_{n-1,0} & Q_{n, 0} \\
Q_{0,1} & Q_{1,1} & \ldots & Q_{n-1,1} & \\
\vdots & & & & \\
& \\
Q_{0, n-1} & Q_{1, n-1} & & \\
Q_{0, n} . & & &
\end{array}
$$

The first, second and third derivative of the points in Casteljau algorithm can be shown with

$$
\begin{gathered}
Q^{\prime}(t)=n_{i}\left(Q_{1, n-1}(t)-Q_{0, n-1}(t)\right), \\
Q^{\prime \prime}(t)=n(n-1)\left(Q_{0, n-2}(t)-2 Q_{1, n-2}(t)+Q_{2, n-2}\right) .
\end{gathered}
$$

Then the equations $Q^{\prime}(0)=\left(Q_{1}-Q_{0}\right), Q_{1}^{\prime}=\left(Q_{n}-Q_{n-1}\right)$ are satisfied. Additionally, the curvature and torsion of Bezier curve with $n$. degree can be found in $[1,3]$.

$$
\begin{aligned}
& \kappa(t)=\frac{(n-1)}{n} \frac{\left\|\left(Q_{1, n-2}(t)-Q_{0, n-2}(t)\right) \times\left(Q_{2, n-2}(t)-Q_{1, n-2}(t)\right)\right\|}{\left\|Q_{1, n-1}(t)-Q_{0, n-1}(t)\right\|^{3}}, \\
& \tau(t)=\frac{(n-2)}{n} \frac{\left\|\left(Q_{1, n-3}(t)-Q_{0, n-3}(t)\right) \times\left(Q_{2, n-3}(t)-Q_{1, n-2}(t)\right)\right\|}{\left\|\left(Q_{1, n-2}(t)-Q_{0, n-2}(t)\right) \times\left(Q_{2, n-2}(t)-Q_{1, n-2}(t)\right)\right\|^{2}} .
\end{aligned}
$$

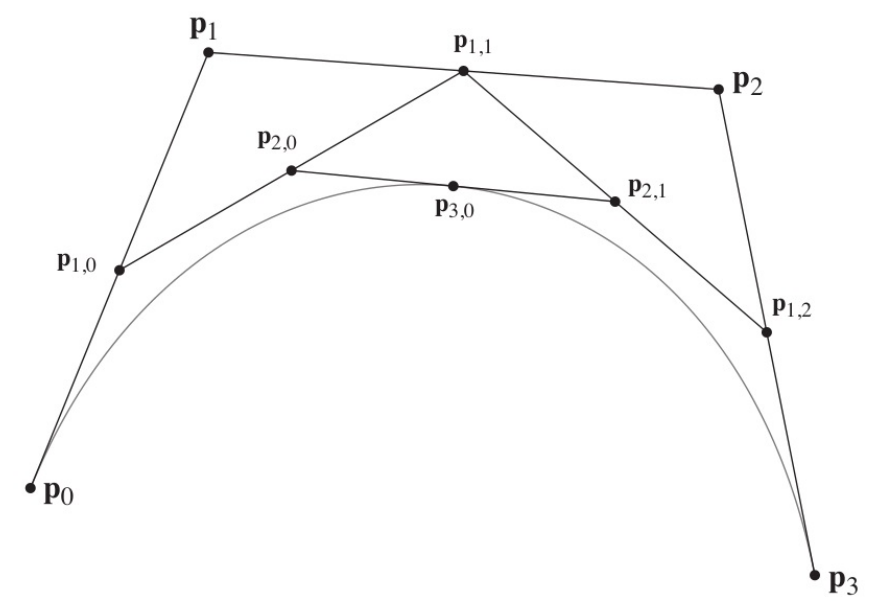

Figure 1. Casteljau Algorithm, [2].

\section{Main Results}

Until now, the Serret-Frenet frame and curvatures of the Bezier curve were known. Presently, a new modified frame of a quadratic Bezier curve will be investigated in this section.

Definition 3.1. Suppose that $Q(t)$ be a quadratic Bezier curve with control points $Q_{0}, Q_{1}, Q_{2}$. A quadratic Bezier curve is defined as

$$
Q(t)=\sum_{i=0}^{2} B_{i, 2}(t) Q_{i}=(1-t)^{2} Q_{0}+2 t(1-t) Q_{1}+t^{2} Q_{2}
$$




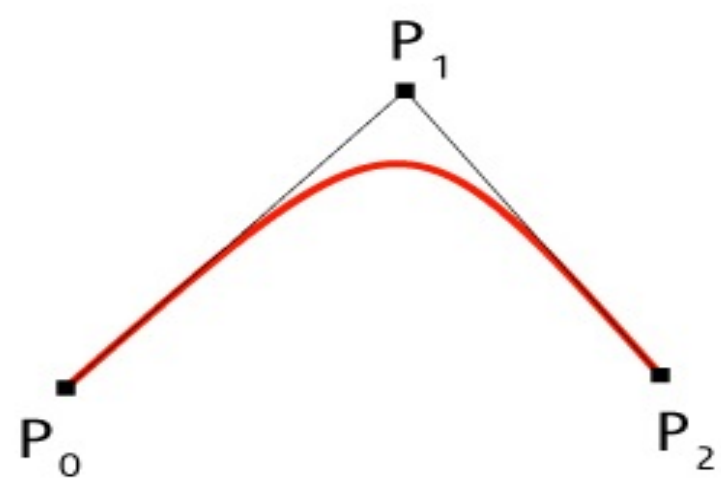

Figure 2. A quadratic Bezier curve.

Theorem 3.2. Let $Q(t)$ be a quadratic Bezier curve. The first order derivative of a quadratic Bezier curve is computed by

$$
Q^{\prime}(t)=2\left((1-t) \overrightarrow{Q_{0} Q_{1}}+t \overrightarrow{Q_{1} Q_{2}}\right)
$$

Proof. Using the Eq.(2.1), we get

$$
\begin{aligned}
Q^{\prime}(t) & =2 \cdot\left(Q_{1,1}(t)-Q_{0,1}(t)\right) \\
& =2 \cdot\left(\left[(1-t) Q_{1}+t Q_{2}\right]-\left[(1-t) Q_{0}+t Q_{1}\right]\right) \\
& =2\left((1-t)\left(Q_{1}-Q_{0}\right)+t\left(Q_{2}-Q_{1}\right)\right) \\
& =2\left((1-t) \overrightarrow{Q_{0} Q_{1}}+t \overrightarrow{Q_{1} Q_{2}}\right) .
\end{aligned}
$$

Hence the proof is completed.

Theorem 3.3. Assume that $Q(t)$ be a quadratic Bezier curve with control points $Q_{0}, Q_{1}, Q_{2}$. The second order derivative of a quadratic Bezier curve is obtained by $Q^{\prime \prime}(t)=2\left(\overrightarrow{Q_{1} Q_{0}}+\overrightarrow{Q_{1} Q_{2}}\right)$.

Proof. Using the Eq.(2.2), we obtain

$$
\begin{aligned}
Q^{\prime \prime}(t) & =2 .\left(Q_{0,0}(t)-2 Q_{1,0}(t)+Q_{2,0}(t)\right)=2 .\left(Q_{0}-2 Q_{1}+Q_{2}\right) \\
& =2\left(\left(Q_{0}-Q_{1}\right)+\left(Q_{2}-Q_{1}\right)\right)=2\left(\overrightarrow{Q_{1} Q_{0}}+\overrightarrow{Q_{1} Q_{2}}\right) .
\end{aligned}
$$

Hence the proof is completed.

Theorem 3.4. Let $Q(t)$ be a quadratic Bezier curve and $Q_{0}, Q_{1}, Q_{2}$ be control points of the quadratic Bezier curve. The Serret-Frenet frame of a quadratic Bezier curve is calculated as

$$
\begin{aligned}
T & =\frac{(1-t) \overrightarrow{Q_{0} Q_{1}}+t \overrightarrow{Q_{1} Q_{2}}}{\left\|(1-t) \overrightarrow{Q_{0} Q_{1}}+t \overrightarrow{Q_{1} Q_{2}}\right\|}, \\
N & =\frac{\left(\overrightarrow{Q_{0} Q_{1}} \times \overrightarrow{Q_{1} Q_{2}}\right) \times\left((1-t) \overrightarrow{Q_{0} Q_{1}}+t \overrightarrow{Q_{1} Q_{2}}\right)}{\left\|\overrightarrow{Q_{0} Q_{1}} \times \overrightarrow{Q_{1} Q_{2}}\right\| \cdot\left\|(1-t) \overrightarrow{Q_{0} Q_{1}}+t \overrightarrow{Q_{1} Q_{2}}\right\|}, \\
B & =\frac{\overrightarrow{Q_{0} Q_{1}} \times \overrightarrow{Q_{1} Q_{2}}}{\left\|\overrightarrow{Q_{0} Q_{1}} \times \overrightarrow{Q_{1} Q_{2}}\right\|} .
\end{aligned}
$$


Proof. Using the Serret-Frenet frame formulas of a non-unit speed curve, the tangent vector of a quadratic Bezier curve is computed by

$$
\begin{aligned}
T & =\frac{Q^{\prime}(t)}{\left\|Q^{\prime}(t)\right\|}=\frac{\left(Q_{1,2-1}(t)-Q_{0,2-1}(t)\right)}{\left\|Q_{1,2-1}(t)-Q_{0,2-1}(t)\right\|}=\frac{\left(Q_{1,1}(t)-Q_{0,1}(t)\right)}{\left\|Q_{1,1}(t)-Q_{0,1}(t)\right\|} \\
& =\frac{2\left(\left((1-t) Q_{1}+t Q_{2}\right)-\left((1-t) Q_{0}+t Q_{1}\right)\right)}{\left\|2\left(\left((1-t) Q_{1}+t Q_{2}\right)-\left((1-t) Q_{0}+t Q_{1}\right)\right)\right\|}=\frac{(1-t) \overrightarrow{Q_{0} Q_{1}}+t \overrightarrow{Q_{1} Q_{2}}}{\left\|(1-t) \overrightarrow{Q_{0} Q_{1}}+t \overrightarrow{Q_{1} Q_{2}}\right\|} .
\end{aligned}
$$

The binormal vector of a quadratic Bezier curve is found by

$$
\begin{aligned}
B & =\frac{Q^{\prime}(t) \times Q^{\prime \prime}(t)}{\left\|Q^{\prime}(t) \times Q^{\prime \prime}(t)\right\|} \\
& =\frac{n^{2}(n-1)\left(Q_{1, n-2}-Q_{0, n-2}\right) \times\left(Q_{2, n-2}-Q_{1, n-2}\right)}{\left\|n^{2}(n-1)\left(Q_{1, n-2}-Q_{0, n-2}\right) \times\left(Q_{2, n-2}-Q_{1, n-2}\right)\right\|} \\
& =\frac{\left(Q_{1,0}-Q_{0,0}\right) \times\left(Q_{2,0}-Q_{1,0}\right)}{\left\|\left(Q_{1,0}-Q_{0,0}\right) \times\left(Q_{2,0}-Q_{1,0}\right)\right\|}=\frac{\left(Q_{1}-Q_{0}\right) \times\left(Q_{2}-Q_{1}\right)}{\left\|\left(Q_{1}-Q_{0}\right) \times\left(Q_{2}-P_{1}\right)\right\|} \\
& =\frac{\overrightarrow{Q_{0} Q_{1}} \times \overrightarrow{Q_{1} Q_{2}}}{\left\|\overrightarrow{Q_{0} Q_{1}} \times \overrightarrow{Q_{1} Q_{2}}\right\|} .
\end{aligned}
$$

The principal normal can be calculated easily from the vector product $N=B \times T$. As a result, from above theorems, the Serret-Frenet frame is obtained. Hence the proof is completed.

Theorem 3.5. Let $Q(t)$ be a quadratic Bezier curve with $Q_{0}, Q_{1}, Q_{2}$ control points. The alternative $\{N, C, W\}$ frame of a quadratic Bezier curve is calculated as

$$
\begin{aligned}
N & =\frac{\left(\overrightarrow{Q_{0} Q_{1}} \times \overrightarrow{Q_{1} Q_{2}}\right) \times\left((1-t) \overrightarrow{Q_{0} Q_{1}}+t \overrightarrow{Q_{1} Q_{2}}\right)}{\left\|\overrightarrow{Q_{0} Q_{1}} \times \overrightarrow{Q_{1} Q_{2}}\right\| \cdot\left\|(1-t) \overrightarrow{Q_{0} Q_{1}}+t \overrightarrow{Q_{1} Q_{2}}\right\|}, \\
C & =-\frac{(1-t) \overrightarrow{Q_{0} Q_{1}}+t \overrightarrow{Q_{1} Q_{2}}}{\left\|(1-t) \overrightarrow{Q_{0} Q_{1}}+t \overrightarrow{Q_{1} Q_{2}}\right\|}, W=\frac{\overrightarrow{Q_{0} Q_{1}} \times \overrightarrow{Q_{1} Q_{2}}}{\left\|\overrightarrow{Q_{0} Q_{1}} \times \overrightarrow{Q_{1} Q_{2}}\right\|} .
\end{aligned}
$$

Proof. The torsion of the quadratic Bezier curve is zero. Thus, using the Serret-Frenet frame formulas of a non-unit speed curve, the alternative frame of a quadratic Bezier curve is computed by

$$
C=\frac{N^{\prime}}{\left\|N^{\prime}\right\|}=\frac{-\kappa T+\tau B}{\sqrt{\kappa^{2}+\tau^{2}}}=\frac{-\kappa T}{\sqrt{\kappa^{2}}}=-T .
$$

Then from the tangent vector, we have

$$
C=-\frac{(1-t) \overrightarrow{Q_{0} Q_{1}}+t \overrightarrow{Q_{1} Q_{2}}}{\left\|(1-t) \overrightarrow{Q_{0} Q_{1}}+t \overrightarrow{Q_{1} Q_{2}}\right\|},
$$

and also because of

$$
W=\frac{\tau T+\kappa B}{\sqrt{\kappa^{2}+\tau^{2}}}=\frac{\kappa B}{\sqrt{\kappa^{2}}}=B,
$$

then we get

$$
W=\frac{\overrightarrow{Q_{0} Q_{1}} \times \overrightarrow{Q_{1} Q_{2}}}{\left\|\overrightarrow{Q_{0} Q_{1}} \times \overrightarrow{Q_{1} Q_{2}}\right\|} .
$$

Hence the proof is completed. 
Corollary 3.6. Let $f$ and $g$ be curvatures of the alternative $\{N, C, W\}$ frame for the quadratic Bezier curve $Q(t)$. The curvatures of the modified frame can be computed as

$$
f=\frac{1 / 4\left\|\overrightarrow{Q_{0} Q_{1}} \times \overrightarrow{Q_{1} Q_{2}}\right\|}{\left\|(1-t) \overrightarrow{Q_{0} Q_{1}}+t \overrightarrow{Q_{1} Q_{2}}\right\|^{3}} \text {, and } g=0 .
$$

Proof. Using the torsion of quadratic Bezier curve is zero. The curvatures will be

$$
f=\sqrt{\kappa^{2}+\tau^{2}}=|\kappa| \text { and } g=\frac{\kappa^{2}\left(\frac{\tau}{\kappa}\right)^{\prime}}{\kappa^{2}+\tau^{2}}=0 .
$$

Thus we obtain

$$
f=|\kappa|=\frac{(2-1)}{2} \frac{\left\|\left(Q_{1,0}-Q_{0,0}\right) \times\left(Q_{2,0}-Q_{1,0}\right)\right\|}{\left\|Q_{1,1}-Q_{0,1}\right\|^{3}}=\frac{1}{4} \frac{\left\|\overrightarrow{Q_{0} Q_{1}} \times \overrightarrow{Q_{1} Q_{2}}\right\|}{\left\|(1-t) \overrightarrow{Q_{0} Q_{1}}+t \overrightarrow{Q_{1} Q_{2}}\right\|^{3}} .
$$

Hence the proof is completed.

Theorem 3.7. Let $Q_{0}, Q_{1}, Q_{2}$ be the control points of a quadratic Bezier curve $Q(t)$. The alternative $N$-Bishop frame of a quadratic Bezier curve is calculated as

$$
\begin{aligned}
N_{1} & =-\cos r \cdot \frac{(1-t) \overrightarrow{Q_{0} Q_{1}}+t \overrightarrow{Q_{1} Q_{2}}}{\left\|(1-t) \overrightarrow{Q_{0} Q_{1}}+t \overrightarrow{Q_{1} Q_{2}}\right\|}-\sin r \cdot \frac{\overrightarrow{Q_{0} Q_{1}} \times \overrightarrow{Q_{1} Q_{2}}}{\left\|\overrightarrow{Q_{0} Q_{1}} \times \overrightarrow{Q_{1} Q_{2}}\right\|}, \\
N_{2} & =-\sin r \cdot \frac{(1-t) \overrightarrow{Q_{0} Q_{1}}+t \overrightarrow{Q_{1} Q_{2}}}{\left\|(1-t) \overrightarrow{Q_{0} Q_{1}}+t \overrightarrow{Q_{1} Q_{2}}\right\|}+\cos r \cdot \frac{\overrightarrow{Q_{0} Q_{1}} \times \overrightarrow{Q_{1} Q_{2}}}{\left\|\overrightarrow{Q_{0} Q_{1}} \times \overrightarrow{Q_{1} Q_{2}}\right\|}, \\
N & =\frac{\left(\overrightarrow{Q_{0} Q_{1}} \times \overrightarrow{Q_{1} Q_{2}}\right) \times\left((1-t) \overrightarrow{Q_{0} Q_{1}}+t \overrightarrow{Q_{1} Q_{2}}\right)}{\left\|\overrightarrow{Q_{0}} \times \overrightarrow{Q_{1}}\right\| \overrightarrow{Q_{2}}\|\cdot\|(1-t) \overrightarrow{Q_{0} Q_{1}}+t \overrightarrow{Q_{1} Q_{2}} \|} .
\end{aligned}
$$

Proof. Using the transformation matrix of N-Bishop and $\{N, C, W\}$ frame $N_{1}=\cos \theta . C-\sin \theta . W, N_{2}=\sin \theta . C+\cos \theta \cdot W$, $N$, where $\theta=\int_{0}^{t} g(t) d t=\int_{0}^{t} 0 d t=r=$ const., the N-Bishop frame can be calculated easily. The curvatures of N-Bishop frame are $k_{1}=f \cdot \cos \theta=|\kappa| \cos r, k_{2}=f \cdot \sin \theta=|\kappa| \sin r$.

\section{NuMERIC EXAMPLes}

Example 4.1. Let be $Q_{0}=(0,0), Q_{1}=(k, l), Q_{2}=(a, 0)$ the control points of a quadratic Bezier curve which a beginning point has located at origin. Then the formula of a quadratic Bezier curve can be written as $Q(t)=\sum_{i=0}^{2} B_{i, 2}(t) Q_{i}=\left(\left(2 t-2 t^{2}\right) k+t^{2} a,\left(2 t-2 t^{2}\right) l\right)$ from using Def. 2.6. The first, second and third derivatives are found by

$$
P^{\prime}(t)=((2-4 t) k+2 t a,(2-4 t) l), P^{\prime \prime}(t)=(-4 k+2 a,-4 l) P^{\prime \prime \prime}(t)=\overrightarrow{0}
$$

Thus the torsion of the quadratic Bezier curve will be zero. The curvature is obtained by

$$
\kappa=\frac{-a l / 4}{\left[(-k-t k+t a-t k)^{2}+(l-2 t l)^{2}\right]^{3 / 2}} .
$$


The Serret-Frenet frame is

$$
\begin{aligned}
T & =\frac{1}{\mu}(2 k-4 t k+2 t a, 2 l-4 t l), \\
N & =\frac{1}{\mu}(4 t l-2 l, 2 k-4 t k+2 t a), \\
B & =(0,0,1),
\end{aligned}
$$

where $\mu=\sqrt{(2 k-4 t k+2 t a)^{2}+(2 l-4 t l)^{2}}$. The $\{N, C, W\}$ frame is found by

$$
\begin{aligned}
N & =\frac{1}{\mu}(4 t l-2 l, 2 k-4 t k+2 t a), \\
C & =-\frac{1}{\mu}(2 k-4 t k+2 t a, 2 l-4 t l), \\
W & =(0,0,1) .
\end{aligned}
$$

As a result, the N-Bishop frame is obtain by

$$
\begin{aligned}
& N_{1}=\left(-\frac{\cos r}{\mu}(2 k-4 t k+2 t a),-\frac{\cos r}{\mu}(2 l-4 t l),-\sin r\right) \\
& N_{2}=\left(-\frac{\sin r}{\mu}(2 k-4 t k+2 t a), \frac{\sin r}{\mu}(2 l-4 t l), \cos r\right) \\
& N=\frac{1}{\mu}(4 t l-2 l, 2 k-4 t k+2 t a) .
\end{aligned}
$$

Example 4.2. Let be $Q_{0}=(0,0), Q_{1}=(0,1), Q_{2}=(1,0)$ the the control points of a quadratic Bezier curve, see in [2]. Then $Q(t)=\sum_{i=0}^{2} B_{i, 2}(t) Q_{i}=\left(t^{2}, 2 t-2 t^{2}\right)$. Thus the torsion of the quadratic Bezier curve will be zero. The curvature is obtained by $\kappa=\frac{-1 / 4}{\left[t^{2}+(1-2 t)^{2}\right]^{3 / 2}}$. The Serret-Frenet frame is $T=\frac{1}{\mu}(2 t, 2-4 t), N=\frac{1}{\mu}(4 t-2,2 t), B=(0,0,1)$ where $\mu=\sqrt{2 t^{2}+(2-4 t)^{2}}$. The modifed frame is calculated by $C=-\frac{1}{\mu}(2 t, 2-4 t), W=(0,0,1), N=\frac{1}{\mu}(4 t-2,2 t)$. The N-Bishop frame is obtained as

$$
N_{1}=\left(-\frac{2 \cos r}{\mu} t,-\frac{\cos r}{\mu}(2-4 t),-\sin r\right), \text { and } N_{2}=\left(-\frac{2 \sin r}{\mu} t, \frac{\sin r}{\mu}(2-4 t), \cos r\right), N=\frac{1}{\mu}(4 t-2,2 t) .
$$

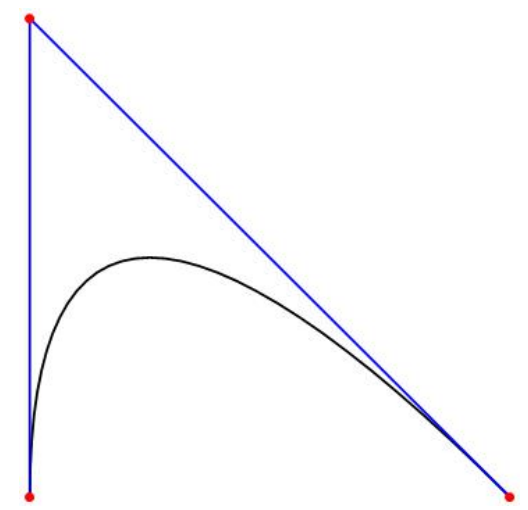

Figure 3. A Mathematica output of the Example 4.2.

\section{Conclusion}

The concept of a quadratic Bezier curve method is one of the important methods for CAGD. In our paper, some properties of a quadratic Bezier curve have been calculated for according to a new alternative frame called N-Bishop frame. Some results of the N-Bishop frame have the abilities to be computed an alternative curvature and torsion for all points of the quadratic Bezier curves. Thus we think that our study will guide other researchers. 


\section{ACKNOWLEDGEMENT}

The paper has been presented in ICPAM-Van 2018, and only the abstract of the article has been published in a conference proceeding book. The authors of this paper would like to express their sincere appreciation to the anonymous referees for their valuable comments and suggestions which have led to an improvement in the presentation of the paper.

\section{CONFLicts OF INTEREST}

The authors declare that there are no conflicts of interest regarding the publication of this article.

\section{REFERENCES}

[1] Bishop, R. L. There is more than one way to frame a curve, The American Mathematical Monthly, 82(3)(1975), 246-251. 1, 2, 2

[2] Farin, G., A history of curves and surfaces, Handbook of Computer Aided Geometric Design, 2002. 1, 2, 2, 4

[3] Floater, M.S., Derivatives of rational Bézier curves, Computer Aided Geometric Design, 9(3)(1991), 161-174. 1, 2

[4] İncesu, M., Gürsoy, O., The principal forms and curvatures on Bezier curves, XVII National Mathematics Symposium, Abant İzzet Baysal University, (2004), 146-157. 1, 2

[5] Keskin, O., Yayli, Y., An application of N-Bishop frame to spherical im-ages for direction curves, International Journal of Geometric Methods in Modern Physics, 14(11)(2017). 1, 2

[6] Marsh, D., Applied geometry for computer graphics and CAD, Springer-Verlag, Berlin, 2005. 1, 2

[7] Samanci, H. K., Celik, S., İncesu, M. , The Bishop Frame of Bezier Curves, Life Science Journal, 12(6)(2015). 1, 2

[8] Sapidis, N.S., Frey, W.H., Controlling the curvature of a quadratic Bezier curve, Computer Aided Geometric Design, 9(1992), 85-91. 1

[9] Scofield, P.D., Curves of constant precession, The American mathematical monthly, 102(6)(1995), 531-537. 1, 2

[10] Uzunoğlu, B., Gök, İ., Yaylı, Y., A new approach to curves of constant precession, Applied Mathematics and Computation, 275(2016), 317323. 1,2

[11] Yılmaz, S., Turgut, M., A new version of Bishop frame and an application to spherical images, Journal of Mathematical Analysis and Applications, 371(2)(2010), 764-776. 1, 2

[12] Yılmaz, S., Özyılmaz, E., Turgut, M., New spherical indicatrices and their characterizations, An. St. Univ. Ovidius Constanta, 18(2)(2010), 337-354. 1 